\title{
Epigenetics: Impacts on Disease Susceptibility and Pharmacotherapy
}

\author{
Abdullahi Rabiu Abubakar ${ }^{1}$ and Mainul Haque ${ }^{2^{*}}$ \\ ${ }^{1}$ Assistant Lecturer, Faculty of Pharmaceutical Sciences, Bayero University Kano, 700231 Kano, Nigeria. \\ ${ }^{2}$ Professor, the Unit of Pharmacology, Faculty of Medicine and Defense Health, National Defense University of Malaysia, Kem Sungai \\ Besi, 57000 Kuala Lumpur, Malaysia.
}

\begin{abstract}
Deoxyribonucleic acid (DNA) is a hereditary material comprising of all the genetic information in humans and almost all other living creatures. It is made up of subunits called genes, which have four nucleotides. MicroRNAs comprises of the short noncoding RNA. They are non-protein coding with about 18-22 nucleotides which control gene expression and activities post-transcriptionally. Notably, the whole DNA structure usually coiled itself around a protein called histone moiety. Consequently, any addition of methyl, ethyl, phosphate, Ubiquitin or small ubiquitin-related modifier either directly on the DNA itself or the histone molecule will ultimately change its configuration and stability leading to the epigenetic modifications. Usually, epigenetic changes manifest phenotypically without changing the actual gene sequence. MicroRNAs also play a vital role in the control of the expression epigenetic regulators, such as DNA methyltransferase (DNMT), histone deacetylase, and Polycomb group genes. The implication of this may significantly affect the normal function of an organism and perhaps contribute to the wide-range of disease aetiology. As such, epigenetics linked variations and diseases are inheritable. The landmark achievement in pharmacotherapy involves the possibility of silencing the oncogenes that are cancer provoking genes and possible reactivation of cancer suppression genes that battle with and repress the tumour cells proliferation. Besides these, more genes that are linked to other diseases apart from cancer can be identified, silenced or activated using drugs or diets in order to treat the disease aetiology. This appraisal is to discuss the role of epigenetic changes in the disease susceptibility and drug treatment.
\end{abstract}

Key words: Epigenetics, Gene-methylation, Histone, Methyl-group, Cancer, Pharmacotherapy.

\section{INTRODUCTION}

Epigenetic changes explain how modified DNA or modified proteins (histone moiety) associated DNA are transferred to the newborn during cellular replication and bring about variations in the new-born characteristics. Epigenetics modify the expression of allele phenotypically without altering their genetic compositions. ${ }^{1-5}$ in addition, epigenetic changes determine which gene is to be expressed and which one to remained dormant or silence. However, unlike genetic polymorphism that alters the gene sequence in DNA, epigenetic modifications affect only phenotypic expressions. $^{2-6}$ During epigenetic changes, genetic imprinting takes place, a condition in which, if the allele inherited from the father is imprinted (silenced), only the allele from the mother would be expressed phenotypically in the new offspring..$^{1-5}$ Generally, epigenetic process occurs via two mechanisms. Firstly, it involves the addition of methyl groups called genetic flags or marker to the genes on DNA strands. The methyl groups attached to the genes will obstruct further communication or exchange of information between DNA within the nucleus and the surrounding cytoplasm. ${ }^{2-5}$ Secondly, DNA molecule normally winds
Submission Date : 02-12-2015

Revision Date : 29-02-2016 Accepted Date : 02-03-2016

DOI: 10.5530/ijper.50.3.1

Correspondence:

Dr Mainul Haque

Professor of the Unit of

Pharmacology,

Faculty of Medicine and

Defense Health,

National Defense University of Malaysia,

Kem Sungai Besi, 57000 Kuala Lumpur, Malaysia. Land Line: +60390512523 (Office), +60361795871 (Home).

Cell Phone: +60109265543.

Email: runurono@gmail.com 
itself around nuclear protein known as histone. Histone protein has five different classes namely, H1, H2A, $\mathrm{H} 2 \mathrm{~B}, \mathrm{H} 3$, and $\mathrm{H} 4$; H1 is loosely bound to chromatin hence easily removed. Thus, $\mathrm{H} 2 \mathrm{~A}, \mathrm{H} 2 \mathrm{~B}, \mathrm{H} 3$, and $\mathrm{H} 4$ are the major groups of histone protein. ${ }^{7}$ Attachment of methyl groups around the histone moiety will block the communication between the genes coiled around it and the entire cytoplasm..$^{3-6,8-10}$ Consequently, this will cut the exchange of information between DNA and the entire cell structure leading to the gene silencing (epigenetic variations). During DNA replication, the methylated genes and those wound around methylated histone are copied or replicated together with their markers and transmitted to the offspring owing to epigenetic changes. ${ }^{2-6,8}$ The phenomenon of gene methylation or silencing occurs by adding methyl groups to the gene and turned it off making it dormant. On the other hand, the addition of any substance that could remove the methyl group will turn that gene on making them active.,3,5,9,11-14 Environmental variables such as exposure to extreme heat, cold, harsh chemicals, radiations, chain smoking or eating some classes of food or drinks (that donates methyl groups) contributes to epigenetics modifications that could be inherited from one generation to another. ${ }^{1,5,8,9,14}$ Notably, epigenetic changes involve chromosome inactivation, genetic imprinting, and centromeres and so on. It is also qualified based on the three parameters as follows: it is usually not based on DNA sequence, it must be heritable variations, and it must be associated with chromosome. ${ }^{2,715}$ Therefore, epigenetic modifications affect only the phenotypic expression of the genome unlike gene mutation and genetic polymorphism that affects the genotypic expression of a genome., ${ }^{2,15}$ Experimentally, epigenetic changes were demonstrated as a violation of the law of mass action by DNA molecule in that two genetically identical DNA molecules in terms of gene composition and sequence behave differently. ${ }^{2,715}$ An example of epigenetic variations includes splicing of female specific Sex-lethal in Drosophila fly to produce more active sex-lethal splicing factor, bacterial expression of LacY, lactose permease, duplication of gene copies in the plant, and somatic mosaicism due to $\mathrm{X}$ chromosome inactivation. ${ }^{15}$

\section{Definition of Terms}

Epigenetic Changes: is an inheritable change in the gene expression that alters the physical appearance or biological function of DNA without any modifications in DNA structure or sequence. In general, methylation of genes turns them off while removal of these methyl groups will turn the genes on. ${ }^{1-5,8,9,11}$
Genetic Polymorphism: refers to the changes in DNA original nucleotide sequence. Each gene on a DNA molecule comprised of nucleotides arranged in a specific manner. Any change in the arrangement of these nucleotides will distort the information contained in that gene. Genetic polymorphism is a permanent, random and a times irreversible changes that affect a population of similar cells or genes. ${ }^{4,15}$

Gene Mutation: is a change in the nucleotide sequence of RNA or DNA molecule due to the replication error or lesion from exogenous or endogenous mutagens or as a result of deletion or insertion of the base pair on a DNA segment. ${ }^{7}$ Unlike genetic polymorphisms, gene mutation affects the only single cell. This single cell may multiply to a population of cells, giving rise to genetic polymorphism. ${ }^{15,16}$

Gene Expression: is a process of translating the encoded message in a gene is into a protein which handles the organization of cellular functions. Usually, DNA interacts with ribosome leading to the protein synthesis that is responsible for organism characteristics. However, in non-protein coding genes such as transfer RNA or small nuclear RNA genes, the product is a functional RNA. . $-5,8,9,11,16^{-16}$

Gene Activation: this refers to the processes involved in reactivation of the dormant genes to make them function again. Once the gene is revived, and the DNA is replicated, the traits contained in the parent DNA will be transmitted to the offspring. ${ }^{17}$ Furthermore, the DNA containing those genes will interact with the ribosome in the surrounding nucleus causing protein synthesis. Through this pathway, the encoded message in those genes will be translated into action. ${ }^{17}$ Gene activation includes removal of a methyl group from the gene, a reaction catalysed by DNA demethylase; removal of a methyl group from histone moiety, a reaction catalysed by histone demethylase; and acetylation of histone, a reaction catalysed by histone acetyltransferase. ${ }^{2,3,6,9,17}$

Gene Silencing: includes all the pathways through which genes are off or become dormant. All the information carried or encoded by the dormant genes will not be seen in offspring. ${ }^{17}$ Gene silencing involves methylation of the gene, a reaction catalysed by DNMT; methylation of histone, a reaction catalysed by histone methyltransferase; and the removal of ethyl group from histone moiety, a reaction catalysed by histone deacetylase. , $^{2,3,6,9,17}$

\section{EPIGENETIC VARIATIONS}

A recent study discovered that women present at the scene of bombast develop post-traumatic disorder owing to the low level of stress hormone in saliva. 
The decline in stress hormone can also be detected in their new-born babies. ${ }^{8}$ Also, children born to parents who had enough food before puberty are more likely to suffer from diabetes. ${ }^{8,13}$ Equally babies that inherited deleted section of chromosome 15 from the mother may experience Angelman syndrome, whereas babies that inherited similar chromosome from the father are likely to develop Prader Will's syndrome. ${ }^{1,8}$ In addition, children born to a mother who starved during puberty have a higher risk of developing glucose intolerance, obesity, coronary heart disease, breast cancer, drug addiction and schizophrenia., ${ }^{8,10}$ Unlike genetic polymorphism and genetic mutation, epigenetic changes are preventable and reversible and can be treated with drugs and diets. ${ }^{2,8,13}$ During cell division, methionine from diet supply methyl group that attached to the pair of the chromosome at Cytosine-Phosphate-Guanine (CPG) axis by enzyme DNMT. ${ }^{1,2,7,8,13,18-19}$ At this point, the methylated chromosome strand attached itself to the unmethylated strand to form pairs. The DNMT enzyme subsequently adds a methyl group to the unmethylated CPG Island in a daughter DNA., 1,2,7,8,13,18,19 The daughter DNA usually winds itself around a histone protein to produce chromatin. Processes such as methylation, acetylation, phosphorylation, ubiquitination or sumoylation of chromatin around histone may give rise to epigenetic changes. Once DNA is methylated it will form a densely packed structure that modify the DNA itself and impair gene expression by turning it off. ${ }^{2,6,11,12}$ Nonetheless, apart from deletion of these methyl groups, attachment of ethyl group comprising of two carbons, one oxygen and three hydrogens around the histone moiety could turn these genes on. This phenomenon is called flipping the gene switch., ${ }^{2,6,13}$

\section{Epigenetics and Genome Wide Associations Study (GWAS)}

GWAS is usually carried out to establish genomic imprinting (gene methylation) and genetic polymorphism and evaluate the role of epigenetic modification and genetic polymorphism in the disease prevalence. ${ }^{4,15}$ Several procedures were employed in the determination of epigenetic changes. The DNA of the patients and that of the control groups or the DNA of the mother and father in a study of inheritance are first isolated from their blood sample using polymerase chain reaction (PCR) technique. ${ }^{4,15,19}$ The next step is the expression of the gene responsible for a particular disease aetiology from the DNA. Additionally, a specific locus of at least three different alleles are selected from both experiment and control groups and both mother and father., ${ }^{4,15}$ Each allele should be considered at least twice to ensure effective representation of all the genotypes. The expression of the chosen alleles in the diseased group and that of the offspring can be done through the use of the mRNA-seq stored database. ${ }^{4,15,19}$ Subsequently, the genetic imprinting in the diseased individual and the abnormal offspring could be evaluated from the different locus of the selected alleles by comparing them with normal gene expression in the normal individual or normal offspring obtained from mRNA-seq store database. ${ }^{4,15,16,19}$ The importance of epigenetics variation with regards to inheritance and genomic evolution was further clarify by GWAS. It was evident that about $50 \%$ of the genomes of the majority of metazoans are highly polymorphic and have contributed to population evolution. ${ }^{1,15}$ Unfortunately, GWAS, population genetics studies and quantitative trait loci were unable to account and explain these changes. Therefore, it can be suggested that apart from genetic polymorphism there is possibly some changes in the genome of an organism contributing to genetic variation and population evolution. ${ }^{15}$ The GWAS research analyzes the heterochromatin that consists of hundred million copies of gene or sequences. Also, the heterochromatin is made up of a densely or tightly packed DNA that form repetitive sequences as indicated by gene expression. ${ }^{7,15}$ Furthermore, any addition of methyl group on the gene or the histone molecule will modify the DNA structure, and may alter the genome orientation and stability and bring about epigenetic variations. For these reasons, the heterochromatin structure now became complex, as such it cannot be easily analysed or read using a simple technique that is directed at the whole genome. ${ }^{2,6,15}$ It was suggested that a genetic variation in the genome conformation does not affect gene sequence but the number of sequences (gene copies) and their configurations. This phenomenon is referred to as copy number polymorphism. Sometimes, epigenetic variations and genetic polymorphism are interrelated; one can hardly explain them as mutually exclusive. ${ }^{2,6,15}$ The histone molecule to which DNA is wrapped is responsible for stabilizing the genome structure. Any distortion in the protein molecule by epigenetic markers (gene or histone methylation) will affect the heterochromatin or genome configuration. ${ }^{7}$ There is strong indication that disruption of gene sequence-toprotein in heterochromatin will result to distortion of gene expression throughout the genome and perhaps give rise to genetic polymorphism. ${ }^{2,6,15}$ It can be inferred that epigenetic changes and genetic polymorphism may overlap in other words they are not mutually exclusive. ${ }^{7}$ On the other hand, euchromatin is lightly packed chromatin or group DNA/RNA/protein that consists of genes in 
a variety of sequences that are mostly under constant replication. ${ }^{2,6,15}$

\section{Epigenetics and Sources Methyl Group}

Dietary choline can be found in liver, wheat germ and eggs, and it serves as a major source of the methyl group in a diet. ${ }^{9,20}$ The choline usually donates methyl groups during the production of the various metabolites. The pathways include the formation of betaine, methionine from homocysteine, and other choline metabolites. ${ }^{9,20}$ Besides this, choline is also important in the biosynthesis of acetylcholine, bioactive phospholipids, and cell membranes. In general, dietary choline is very essential, although the major portion of dietary choline is produced via endogenous denovo synthesis., ${ }^{20}$ The denovo production of choline occurs via synthesis of its intermediary phosphatidylcholine catalysed by phosphatidylethanolamine $\mathrm{N}$-methyltransferase (PEMT) in the liver. ${ }^{2,20}$ It is imperative to note that Vitamin $\mathrm{B}_{12}$ and folic acid are also essential in the synthesis of methionine, a methyl group donor obtained from choline. This reaction is catalyzed by methionine synthase. ${ }^{2,20}$ Overall, the deficiency of dietary choline, vitamin $\mathrm{B}_{12}$, and folic acid and or single nucleotide polymorphism (SNPs) in enzymes involve in this pathway such as PEMT, methionine synthase may produce an elevated plasma level of homocysteine leading to fatty liver, cell damage, altered brain structure and low memory in children., ${ }^{9,20}$ The dietary choline requirements usually increase in pregnant women due to its utilization in milk production and use by the fetus in brain and memory development. Interestingly, premenopausal women may not suffer dietary choline deficiency because once the choline is inadequate, oestrogen response element (ERE) will stimulate upward regulation of PEMT genes leading to more synthesis of choline endogenously. ${ }^{2,920}$ Nonetheless, children born to choline deficient mothers may experience a substantial alteration in brain anatomy and low memory. ${ }^{2,920}$ Notably, while a large number of people develop diseases or genetic defects due to deficiency of dietary choline others do not. Thus, various researchers suggested that epigenetic variation and or genetic polymorphism are perhaps responsible for individual variation in the dietary choline requirements. ${ }^{2,20}$ Furthermore, choline or its metabolites donate methyl groups for DNA methylation leading to genomic imprinting, gene expression, gene transcription and gene stability., ${ }^{2,20}$ Choline deficiency may cause a decrease in methylation of cyclin-dependent kinase inhibitor 3 (CDKN3) enzyme gene promoter leading to overexpression. Eventually, this may inhibit neurogenesis (brain cell proliferation) and cause birth defects. ${ }^{2,9,20}$ This problem, if early detected could be resolved by feeding the mother with choline-rich diet to promote methylation of CDKN3 gene, silenced it and enhance neurogenesis. It was suggested that epigenetic modifications due to high choline as methyl group source is important in foetal development and can be used to prevent organ damage. ${ }^{2,920}$

\section{Epigenetics and DNMT Enzymes}

DNMT enzymes catalyze the genomic imprinting or the gene methylation during gene expression. About four different categories of this enzyme were identified in mammals namely DNMT1, DNMT2, DNMT3A, and DNMT3B. ${ }^{2,7,11,18}$ It was suggested that SNPs in the genes responsible for the expression of these enzymes perhaps affect their role in methylation of mutant genes. Consequently, the role of mutant genes in the pathogenesis of cancer may be altered. ${ }^{2,11,13,18,21}$ In other words, genetic polymorphism of DNMT enzymes may affect their role in epigenetic modifications via gene methylation. Therefore, genetic polymorphisms and epigenetic variations may contribute simultaneously to the aetiology of cancer. ${ }^{2,11,13,18,21}$ The progress of cancer depends on whether the oncogenes or cancer suppressing genes is methylated, or their methylation is inhibited and vice versa. In addition, cancer and autoimmune disease are believed to occur when there is hypermethylation of promoter CPG linked bases and hypomethylation of another region of the genome., ${ }^{2,914,18,21}$ Research has revealed that epigenetic modifications such as hypomethylation of CPG island of ERE will turn the genes on and suppress cancer growth. In other words cancer growth is under estrogen control. However, hypermethylation will turn the genes off and favor cancer growth. ${ }^{1,7,18,20,21}$ In breast cancer, the activities of DNMT1 is controlled by MicroRNA. Thus, phosphorylation and downregulation of MicroRNA will turn the ERE genes off and enhance tumorigenesis, although the exact mechanism is not yet understood. ${ }^{7,10,16,18,21}$ During foetal life, denovo methylation of DNA in the embryo is catalysed by DNMT3A and DNMT3B while maintenance methylation is controlled by DNMT1., 2, $7,16,18,21$

\section{Epigenetics and Histone Deacetylase (HDAC) Enzymes}

Histone deacetylase enzymes remove the acetyl moiety attached to the histone protein. The acetyl groups play a vital role in gene expression and stability. As such their removal will distort the genome conformity and inhibit the gene expression., ${ }^{2,67}$ These enzymes are made up of about 18 different groups in human cells which are divided into two families: ${ }^{6,7}$ 
(1). Zinc-dependent groups:

(a) Class 1: they use histone protein as substrate and they are located in the cell nucleus. Examples include HDACs 1, 2, 3 and 8

(b) Class IIa and IIb: they are located within the cytoplasm, but times they can move into the nucleus and use both histone and non-histone protein as a substrate. Examples are HDAC 4, 5, 6, 7, 9 and 10

(c) Class IV: this possesses all the properties of class I and II enzymes, they are found in both nucleus and in the cytoplasm. Example is HDAC 11

(2). Zinc independent but NADH-dependent groups: they are found in mammalian cells and use nonhistone protein as a substrate. They are also known as CLASS III of sirtuins.

The role of these enzymes is to controls the whole genome activities. Meanwhile, the primary role of HDAC enzymes is to remove the acetyl groups attached to the histone moiety and eventually suppress the gene expression. ${ }^{2,67}$ The actions of these enzymes that lead to gene silencing have been implicated in many disease pathologies. A class of drugs called HDAC inhibitors blocks these enzymes and favour further acetylation of histone protein. ${ }^{26,7}$ These drugs will restore the gene expression to normal without altering the DNA sequence. They are useful in the treatment of epigenetically induced disease including neoplasm and neurodisorders, and they are also called epigenetic modifiers. ${ }^{2,6,7}$

\section{Gene Methylation Experiment}

The epigenetic modification could be identified experimentally by determining the extent of genome methylation of an aging individual or patient suffering from a particular disease. Effective monitoring of DNMT enzymes activities will helps in early diagnosis of cancer. ${ }^{3,18}$ The existing procedure employed in monitoring the gene methylation involves the use of fluorescence, radioactivity, antibodies and obtrusive instrumentation that are cumbersome. Conversely, electrochemical methods offer high convenience, sensitivity, easy to apply and reproduce. $^{18}$ A label-free electrochemical platform is used in detecting the activity of DNMT1 enzymes in crude lysates, from both cultured human colorectal cancer cells and colorectal tissue biopsy samples. ${ }^{18}$ It involves the use of electrodes on which the lysates sample is applied, followed by restriction enzymes that detect the presence of methylation on that site. Once the DNA is methylated, it will be amplified by the restriction enzymes leading to the production of electrochemical signals. ${ }^{18}$ But, if the DNA is not methylated in the lysate sample by the active DNMT1, the restriction enzymes will cleave the DNA and significantly diminish the electrochemical signal from the DNA. ${ }^{18}$ Specifically, the DNA used is low-density DNA monolayers formed using a 15-pin setup to prevent clogging. Both the primary and the secondary electrodes are made up of gold rod embedded in Teflon. ${ }^{18}$ The electrodes surfaces were polished, and monolayers were formed using ethanolic solution and of $1 \mathrm{M}$ 12-azidododecane-1-thiol $\left(\mathrm{C}_{12}\right.$ thilazide) and $1 \mathrm{M}$ 11-mercaptoundecylphosphoric acid (Sigma-Aldrich). ${ }^{18}$ In addition, negative potential ( $-350 \mathrm{mV}$ for 25 minutes) is applied to the secondary electrode and in the presence of restriction enzymes and Tris buffer $\left(100 \mathrm{mM} \mathrm{KCl}, 2.5 \mathrm{mM} \mathrm{MgCl}_{2}\right.$ and 1 $\mathrm{mM} \mathrm{CaCl}_{2}$ at $\mathrm{PH}$ 7.6). ${ }^{18}$ Based on the type of monolayer formed on the electrode, multiple sequences of DNA with different methylation state will attach themselves to the different electrode. The electrochemical signals are measured using Amperometry. ${ }^{18}$ The measurement is usually done by treating the electrodes with active DNMT1 enzymes in pure form or as part of the lysate mixture. On one hand, presence of hemimethylated DNA as substrate and SAM as a cofactor, and application of DNMT1 protein will turn the signal from the electrode 'ON' and remains protected..$^{18}$ On the other hand, in the absence of gene methylation, the restriction enzymes will turn the signal from the electrodes 'OFF' signifying the low level of methylation. ${ }^{18}$ It is important to note that using this method, human biopsy tissue samples can be differentiated from the adjacent normal tissue. The biopsy tumour tissues or cancer cell line are usually purchased and stored using liquid nitrogen at $-80^{\circ} \mathrm{C} .{ }^{18}$ The DNA obtained from the tumour cells normally display signal protection due to hypermethylation whereas the normal cells show signal restriction due to the hypomethylation. ${ }^{18}$ Inferentially, tumour cells from both cultured colorectal carcinoma cells and tissue biopsy can be distinguished from the normal cells through electrochemical DNMT1 measurement. An early method of cancer screening and inform treatment options can be actualized through monitoring the methyltransferase activity in a crude tissue lysate. ${ }^{18}$

The conventional methylation analysis procedure involves setting up criteria and experimental conditions before the experiment begins. ${ }^{3}$ The Primer and all the experimental variables, as well as base line clinical parameters are recorded at the beginning. Once the result obtained is not reproducible or unsatisfactory, the initial conditions can be altered, and the test reruns again. ${ }^{3}$ The procedure begins with the extraction of DNA from Buffy coat of $7 \mathrm{ml}$ of whole blood, followed by quantitative analysis of bisulfite treated DNA by PCR pyrosequencing guidelines. ${ }^{3}$ Also, $500 \mathrm{ng}(25 \mathrm{ng} / \mu \mathrm{L})$ 
of DNA was placed inside enzymes DNA Methylation Gold Kit and treated with $200 \mu \mathrm{L}$ of M-Elution buffer. Methylation assay was carried out using Genomatix Soft Wave by identifying the imprinting promoters. ${ }^{3}$ The assay involves the addition of $25 \mathrm{ng}$ of DNA and water to $50 \mu \mathrm{L}$ of PCR in $25 \mu \mathrm{L}$ of GoTaq Green Master Mix. The mixture of PCR product was purified and then sequenced by pyrosequencing using $0.3 \mu \mathrm{M}$ sequencing primer. $^{3}$ The first step of sequencing is to set the control by verifying the PCR amplifier using standard DNA. Subsequently, the purified PCR is placed on Agarose gel. In the second step, the volunteer's DNA is finally added to the PCR, mixed and analysed to generate data. ${ }^{3}$ The data is subsequently analysed using statistics, and the result can be inferred after statistical analysis and scrutiny by the expert. ${ }^{3}$ A correlation analysis was carried out on the baseline clinical parameters to determine the association between aging and gene methylation. In addition, regression analysis was carried out to evaluate the association between gene methylation and age in a volunteer with an interacting disease condition. ${ }^{3}$ This analysis will extrapolate interaction between baseline clinical parameters (BMI, DBP, SBP, and FBG) and underlying interacting disease condition such as (Diabetes, obstructive pulmonary disease, and hypertension) with respect to age change and DNA methylation. ${ }^{3}$ Categorically, a Wald test of covariance was applied to evaluate the variations due to the effect of age change among the subjects that were not captured by any baseline clinical parameters or disease conditions mentioned earlier., ${ }^{3,22}$

\section{EPIGENETICS AND DISEASE SUSCEPTIBILITY}

Epigenetic variation is responsible for the ability of brain, kidney, heart, lungs, and GIT cells to behave differently despite having similar genetic composition. It also explains the reasons for variations in disease susceptibility by identical twins even though they have similar genetic makeup., ${ }^{2,13}$ The risk of certain diseases such as Alzheimer's disease, diabetes, cancer and obesity can be minimized by thorough gene study of the ancestors and by prescribing appropriate medicine and diets. ${ }^{2,8,13}$ A recent study indicated that the change in sequences alone does not represent the total contribution of genetics on disease prevalence but also the epigenetic modifications. ${ }^{2,913,22}$ The human body is regulated by more than 20,000 genes that carry the coded message for the production of insulin, antibodies, heart muscle and other proteins. When biomarkers methylate genes, epigenetic modification will occur turning the gene off and cause the impairment of insulin production and diabetes mellitus. ${ }^{2,13}$ Likewise, gene methylation could result from the diet, drugs, alcohol, smoke and certain chemicals exposed by the individuals that may turn the gene off., ${ }^{213}$ Agouti mouse when fed with extra folic acid, vitamin $\mathrm{B}_{12}$, betaine and choline, the nutrients will provide methyl group which methylate the genes responsible for obesity and cancer turning them off., ${ }^{213,22}$ An offspring born to this mouse is darker, leaner, healthier and less likely to develop cancer or obesity because the oncogenes were silenced by gene methylation. On the other hand, an offspring whose Agouti mother was not placed on similar diet appear with lighter yellow skin, obese and prone to diabetes and cancer. ${ }^{13} \mathrm{~A}$ similar outcome was obtained with Agouti mouse fed with soya bean containing genistein. Notably, through adequate epigenetic study, researchers could reduce the risk of cancer, obesity, diabetes, Alzheimer's disease and more using appropriate drugs and diets. ${ }^{2,8,13}$

\section{(i). Epigenetics and Cancer}

Breast Cancer: cancer is believed to be as a result of gene mutation as well as epigenetic changes. Unlike gene mutations that are difficult to treat and times irreversible, epigenetic variations are reversible and can be treated with drugs and diets. ${ }^{2,8,10,13}$ It was established that epigenetic changes handle $50 \%$ of cancer episode that are preventable and treatable. A genetic mutation involving breast cancer 1 and 2 (BRCA1 \& BRCA2) genes is responsible for cancer affecting breast and it is irreversible. ${ }^{2,8,10,13}$ Nevertheless, using the concept of epigenetics, it is possible to target the BRCA1 and BRCA2 oncogenes, methylate them and turn them off. Accordingly, this will perhaps reduce the risk of breast cancer due to the BRCA1 and BRCA2 gene mutation. ${ }^{13,21}$ Although the silencing of BRCA1 and BRCA2 genes epigenetically is yet to be practiced. It is still perceived as one of the possible measures to breast cancer. A Recent report revealed that BRCA 1 gene mutation causes Angelina Jolly's breast cancer and double mastectomy. ${ }^{13,19}$ In human cells, a balance exists between cancerous genes (oncogenes) which provoke the proliferation of tumour cells and cancer suppressing genes that slow down the growth of cancer cells. ${ }^{2,13,14}$ Cancer may manifest when more oncogenes are expressed due to lack of methylation or presence of ethylation or due to the turning off of tumour suppressing genes. ${ }^{2,13,14}$ Ordinarily, nutrients like genistein in soya beans, sulforaphane in vegetables, curcumin in turmeric, resveratrol in grapes may inhibit DNMT enzymes responsible for gene methylation in a test tube. ${ }^{13}$ Depending whether oncogenes or cancer suppressing genes is methylated, the relative risk of developing cancer will either decrease or increase. Meanwhile, it was found that hypermethylation of tumour 
suppression genes make them dormant and increase the risk of cancer. ${ }^{13,14}$

Epigenetic Mechanism: generally, epigenetic mechanism involves four distinct stages such as: ${ }^{7,19}$

(i) DNA methylation which is usually cause gene silencing

(ii) Covalent histone modification which usually yield gene activation or silencing depending upon which residues are modified

(iii) Non-covalent nucleosome positioning and histone variants that result in either gene silencing or activation by modulating chromatin accessibility

(iv) MicroRNAs modification that strictly produce gene silencing. ${ }^{7,19}$

Epigenetic Mechanism in Cancer: the pathophysiology of tumour involve either of the following stages or combination of both. ${ }^{7,19}$

(a) Hypermethylation of genomic C-P-G Island on a tumour suppressor gene leading to its silencing and increase risk of cancer

(b) Hypomethylation of oncogenes leading to their overexpression and increase in risk of cancer

(c) Histone modification which alter the chromatin structure and disrupt transmission of genetic information and promote cancer growth. ${ }^{7,19}$

\section{(ii). Epigenetics and Alzheimer's disease}

Epigenetics plays a vital role in cognitive impairment and dementia in Alzheimer's disease. In a study involving two identical twins, one is Alzheimer's disease patient while the other one is healthy, it was discovered that the genes in the Alzheimer's patient are less methylated and more turned on than that of the healthy twins. ${ }^{2,13} \mathrm{~A}$ similar outcome was obtained when 20 Alzheimer's patients versus 20 healthy patients were studied. However, it is not yet established whether the loss of methylation due to the epigenetic marker causes dementia., ${ }^{2,13}$ Experiment in a test tube revealed that certain chemicals result in the formation of Tangles and Plaques in the brain that led to Alzheimer's disease. ${ }^{13}$ Once the absence of methylation in Alzheimer's patient could be detected as early as possible, drugs and diet that could enhance gene methylation would possibly, prevent, slow or postpone the disease if prescribed. ${ }^{2,8,13,14}$ Conversely, it remains ambiguous whether the drugs or diet will add the methyl groups to oncogenes or cancer suppressing genes.

\section{(iii). Epigenetics and Epilepsy}

Epigenetics plays a vital role in epileptogenesis in a patient with structural brain lesion like hippocampal sclerosis. Usually, DNA methylation or histone alteration leads to modification of chromatin and gene expression. ${ }^{2,3,6,11,12}$ Epigenetic is involved in the control of the central nervous system and memory regulation, as such it contributes to the aetiology of neurologic disorders like epilepsy, schizophrenia, and spinal muscular atrophy. Equally, these disorders contribute to epigenetics changes and vice versa. ${ }^{2,3,11,12}$ The effect of epigenetics on epileptogenesis is believed to occur via regulation of genes responsible for signal transduction, inflammation, cell metabolism, ion transport, synaptic transmission, and stress. ${ }^{11,12}$ Epigenetic variations such as increased expression of neuron-restrictive silencing factor were identified in pilocarpine induced status epilepticus. ${ }^{11,12}$ Secondly, high level of DNA promoter methylation was found in temporal lobe epilepsy. Thirdly, increase in Reelin DNA promoter methylation in epilepsy was recognized during bisulfite sequencing of DNA obtained from dentate gyrus. ${ }^{22}$ Perhaps this confirmed the roles and contribution of epigenetics in neuronal diseases. ${ }^{11,12}$ Once Reelin is silenced epigenetically, granule cell dispersion will be emerged causing epilepsy. The role of epigenetics is also evident by amplification of expression of CREB-binding protein in kainic acid induced status epilepticus and electroconvulsive seizure. ${ }^{11,12} \mathrm{It}$ is imperative to acknowledge that drugs used in the treatment of epilepsy correct the ketogenesis but do not halt the epigenetic processes. Therefore, epigenetic psychotherapy or dietary intervention may be necessary. ${ }^{11,12}$

\section{(iv). Epigenetics and depression}

Recent research explained how hypermethylation of gene encoding serotonin transporter promoter result in its silencing. ${ }^{9,17}$ Also, hypermethylation of this gene will make DNA to become tightly pack into nucleosome around protein. A combination of these will lead to repression of serotonin transporter and cause decrease in the output of serotonin and eventually depression. ${ }^{9,17}$ The possibility of transmission depression from parents to offspring via epigenetic modifications was explained in a research conducted using neuron-specific glucocorticoid receptor (NR3C1) promoter in hippocampi. ${ }^{9}$ The NR3C1 was obtained from suicide victim those with and without a history of sexual and nonsexual assault. The result is compared to the suicide victim from other causes. 'The level of DNA methylation discovered in the subjects with a history of abuse was found to be much higher compared to those without a history of assault and a control group. ${ }^{9}$ Also, there was a decrease in the expression of NR3C1 as measured by the amount of mRNA in the abused suicide victims compared to non-abuse victims and control. This finding has suggested the possibility of inheriting depression via epigenetic changes. ${ }^{916,19}$ In another study, separation of newborn 
mice ten days after their birth can lead to up-regulation of arginine vasopressin (AVP) gene enhancer due to hypomethylation. These modifications may enhance the discharge of corticotrophin releasing hormone and subsequent hyperactivity of hypothalamic pituitary adrenal (HPA) axis. ${ }^{7,918}$ The outcome could manifested as deficits in the forced swim test and step-down avoidance learning tasks. Although, presence of methyl CpGbinding protein 2 (MeCP2) may restore the behavioural modifications resulting from the hypomethylation and recruitment of DNMTs enzymes. ${ }^{7,9,18}$ Brain-derived neurotrophic factor (BDNF) is implicated in the control of DNA methylation. As such, the BDNF genes were found to reduce significantly in the depressed patient due to hyper-methylation. ${ }^{9,19}$ Strong BDNF gene methylation and silencing were identified in a maltreated rat's pulps. Likewise, more DNA methylation was established in the offspring of the maltreated female rats, and they exhibit comparable abusive behaviour as their mothers. Evidently, there is possibility of the inheritance of epigenetic changes from the parent to the offspring. Homocysteine is a methyl donor for DNA methylation, its high concentration in the body may promote hypermethylation, gene silencing and a significant reduction in the gene expression. ${ }^{9}$ Silencing of a gene encoding neurotransmitter such as serotonin will lead to their imbalance or shortage leading to depression, stroke and or heart diseases. ${ }^{9,17}$

\section{(v). Epigenetics and Obesity}

Children whose parents starved during puberty are susceptible to a wide range of diseases as explained earlier. ${ }^{2,13,23}$ Likewise, children born to a mother who had enough to eat during puberty have a higher risk of having obesity and diabetes. ${ }^{2,13,23}$ Additionally, a mother that was starved during conception may gives birth to a new-born with higher weight and larger waist than the new-born to mothers that had malnutrition during the first and second trimester. ${ }^{2,13,23}$ It is because, during the first and second trimester, the insulin-like growth factor II (IGF2) is more expressed, methylated and turned off. In another study it was discovered that in overweight fathers, the IGF2 is less methylated and more turn on, hence give birth to more obese and overweight children., ${ }^{2,23}$ Recent research revealed that methylation of $\mathrm{H} 3 \mathrm{~K} 9$ gene result in silencing of chromatin transcriptionally and also lead to inhibition of PPAR $\alpha$ expression in skeletal muscle. ${ }^{23}$ Mice in which these genes were turned off and which experiance a reduction in $\beta$-oxidation and glycerol release will have decrease in fat oxidation and obesity. ${ }^{23}$ This is event usually accompanied by accumulation of lipid droplets and reduction in expression of uncoupling protein-1 in brown fat. Another appraisal reported the downregulation of insulin-dependent GLUT4 and genes associated with adipogenesis in mice as a result of gene methylation leading to the decrease in fat oxidation and obesity. ${ }^{23}$ Categorically, epigenetic mechanism is involve in the regulation of metabolic genes expression, $\beta$-cell activity, and insulin secretion and release in the islets. ${ }^{23}$ Similarly, GK enzymes promote the regeneration of damaged $\beta$-cells in mice. As such, hypermethylation of the gene encoding for GK enzymes or its deletion may cause hyperglycaemia, hypoinsulinemia and increase in the rate of apoptosis. Consequently, the overall effect could manifest as diabetes and obesity. ${ }^{23}$

\section{(vi). Epigenetics and Aging}

The aging process is describe as the loss of phenotypic plasticity over time. Epigenetic changes such as gene methylation has been recently linked to age-related diseases such Alzheimer's disease, arteriosclerosis, cancer, and diabetes. ${ }^{1-3,11,12}$ As a result of this, a study was conducted using candidate genes for nitric oxide (iNOS), glucocorticoids receptor, coagulating factor 3 (F3), carnitine O-acetyltransferase (CRAT), 8-oxoguanine DNA glycosylase 1 (OGG), interleukin 6 (IL-6), toll-like receptor 2(TLR2), intracellular adhesion molecule, interferon gamma (IFNY) to evaluate the changes in DNA methylation with age., 3 These candidate genes play a role in an inflammatory process, oxidative metabolism and endothelial function that are directly associated with aforementioned age-related diseases., ${ }^{2,3}$ After analyzes it was established that the increase in age is significantly associated with a decrease in the methylation of GCR, TLR2 and iNOS and an increase in the methylation of IFNY, OGG, CRAT, and F3. These may vary among individuals within the population. ${ }^{2,3}$ Another study indicated that there is decrease in the gene methylation in peripheral blood cells and human lymphocytes with an increase in age. ${ }^{3,19}$ Similarly, hypermethylation of IGF2, E-cadherin (urothelial bladder cells), estrogen receptor (human colonic mucosa), p14 (colonic tissue), p16 (gastric epithelial), collagen $\alpha 1$ (periodontal ligament tissue), c-fos (liver tissue) was observed with increase in age. ${ }^{3,19}$ As age increased, the influence of epigenetic changes through biomarkers is better understood by carrying out a cross-sectional study involving a wide range of age volunteers, and also by conducting a longitudinal study to consider the influence of age change on single individual. ${ }^{1-3}$ 


\section{PHARMACOTHERAPY}

Various classes of drugs are used in the treatment of epigenetically induced disorders. The major target is to reactivate the dormant genes by the removal of a methyl group from the genome and histone molecule or by preventing the removal of an acetyl group from the histone moiety. ${ }^{2,69}$ DNAdemethylase removes the methyl group in the genome, and the methyl groups on the histone moiety are removed by histone demethylase. Therefore, induction of these enzymes through diet or pharmacotherapy is likely to improve gene expression., ${ }^{2,69}$ Preferably, the treatment of epigenetically induced disorders especially cancer of any form as well as neuronal disorders is done via inhibition of HDAC enzymes. These class of drugs is called histone deacetylase inhibitors (HDACIs)., 2,6,9,19

\section{Classification of HDAC inhibitors}

These drugs constitute the class of a popular class known epigenetics pharmacotherapy medicines. They include benzamides, hydroxamates, electrophile ketones, cyclic tetrapeptides and depsipeptides and aliphatic compounds. ${ }^{6,7}$ They have a broad spectrum of action in terms of inhibition of various classes of HDAC enzymes especially the hydroxamic acid group. ${ }^{6,7}$ The aliphatic compounds such as valproic acid inhibit class I and II but not IIb while the phenylbutyrate specifically inhibits class I and II HDACs. ${ }^{6}$ Based on the action on zinc-containing catalytic domain of HDACs, the HDAC inhibitors are further classified into the following: ${ }^{6,7}$

1. First generation HDACSi: These includes aliphatic acid compounds (Valproic acid and Phenylbutyrate); Benzamides; Hydroxamic acids (Trichostatin); Electrophile Ketones; Cyclic tetrapeptides and depsipeptides (Trapoxin B and Romidepsin)

2. Second generation HDACIs: Benzamides (Givinostat, Entinostat, and Mocetinostat); Hydroxamic acids (Belinostat, Vorinostat, Panobinostat)

3. Third generation HDACIs: 2-hydroxynaphaldehydes, Nicotinamide and Dihydocoumarin., ${ }^{6,7}$

\section{(I). Treatment of Cancer}

Recently, researchers have broadened their studies on silencing of SLC22A1 gene encoding for organic cation transporter 1 (OCT1) due to gene methylation. ${ }^{14}$ The transporter OCT1 regulate the transportation of some drugs, including anticancer, xenobiotics, nutrients and metabolites in the cells. Silencing of this transporter via gene methylation is believed to make it dormant and unable to express. ${ }^{14}$ Lack of expression of this transporter will prevent a lot of anticancer drugs such as cisplatin, imatinib, irinotecan and paclitaxel that depends on this ion channel from gaining access to tumour cells leading to treatment failure. ${ }^{14}$ However, some of the lipophilic drugs can manage to gain access through passive diffusion. ${ }^{14}$ In hepatocellular carcinoma patient, the OCT1 was found to be hypermethylated, hence not fully expressed in the liver leading to the impairment of cellular transport. ${ }^{14}$ For this reasons, sorafenib which does not require active transport for traveling remains the drug of choice in an advanced hepatocellular carcinoma. ${ }^{14}$ Notably, the SLC22A1 gene encoding for (OCT1) can be used as a marker for predicting the therapeutic drug response. ${ }^{14}$ In another study, it was discovered that hypomethylation of promoter MIRI93A together with RNA 193a-3p transcriptional induction, which target Serine/Argininerich splicing factor 2 (SRSF2) increased hepatocellular cancer cell susceptibility to 5 -flourouracil. ${ }^{14}$ This splicing factor increases tumour cell's sensitivity to 5-flouro uracil anticancer drug. Henceforth, MIRI93A can be used as a marker for therapeutic response to 5-flouro uracil and silencing of this gene will decrease the drug sensitivity and cause treatment failure. ${ }^{14}$ It is important to acknowledge that decitabine which is DNMT inhibitor is approved for pharmacotherapy of solid tumours and lymphomas. ${ }^{14}$ When decitabine is administered together with other classical anticancer drug, it will inhibit gene methylation and promote expression of drug transporter leading to more access to the tumour cells by the anticancer drugs hence increase in efficacy. ${ }^{14}$ Specifically, intake of decitabine could reverse the repression of transporter OCT1 thereby enhancing uptake of cisplatin into hepatocellular cells. Nonetheless, overcoming the issue of chemoresistance with epigenetic pharmacotherapy is yet to be actualized. ${ }^{14}$

Mechanism of Anticancer by HDACIs: in cancer pathology, laboratory investigation revealed that there is an elevation of HDAC1 enzymes in gastric cancer and comparable elevation of HDAC2 and HDAC3 enzymes in colon cancer. ${ }^{6,79}$ Elevation of these enzymes perhaps suggested the significant increase in histone protein deacetylation leading to cancer suppressing genes silencing and optimization of tumour cells proliferations. ${ }^{6,79}$ Thus, inhibition of these enzymes by the drugs indicated in the epigenetic pharmacotherapy will induce histone and non-histone protein acetylation and eventually restore the gene expression and impede cancer growth. ${ }^{6,719}$ Furthermore, hyperacetylation of histone protein will trigger expression of cancer suppressor genes such as p21, p27 and so on and instantaneously depresses the oncogenes such as cyclin $\mathrm{D}$. The concurrent action of HDACIs will halt the tumour growth. ${ }^{6,719}$ Similarly, hyperacetylation of non-histone proteins like p53, pRb, STAT 3 that are useful in cancer cell growth may impair 
their function and ultimately ruin the tumour progression. ${ }^{6,719}$ Alternatively, HDACIs induced acetylation of non-histone proteins such as HIF-1 alpha may diminish the production of Vascular Endothelial Growth Factor or may cause free radical prompted cell expiry by activating the intrinsic and extrinsic apoptotic pathways. ${ }^{6,7}$ Ordinarily, these actions were only possible due to the selective action of some HDACIs and their ability to discriminate between the cancer cell and normal cells. In other words, they can selectively halt the progression of tumour cells while leaving the normal cells intact. ${ }^{6,7}$

\section{(II). Treatment of Alzheimer's disease}

Alzheimer's disease is a neurodegenerative disease that is perhaps due to the silencing of the gene responsible for the control of neurogenesis. Research has shown that the process of memory formation and cognitive function may be impaired by repressing the gene involved in synaptic plasticity following the gene methylation or histone modification. ${ }^{6,7}$ Thus, drugs such as HDACIs may correct the histone modification and treat the cognitive impairment. Consequently, HDACIs have potential to be used in the pharmacotherapy of psychiatric diseases and neuro-genitive disorders. ${ }^{6}, 7$ In the pathophysiology of Alzheimer's disease, there is hyperphosphorylation of Tau protein leading to the generation of neurofibrillary tangles and extracellular store of $\beta$-amyloid. The situation has prompted the neuron degeneration and sign and symptoms of Alzheimer's disease. ${ }^{6,7}$ A nicotinamide is a class III HDACI which act by restoring certain cognitive impairments in transgenic mouse model suffering from Alzheimer's disease. ${ }^{6}$ In addition, valproic acid is another drug indicated for the treatment of Alzheimer's disease; it suppresses the formation of $\beta$-amyloid in the brain of Alzheimer's disease transgenic mouse model. ${ }^{6}$ Also It was found have neuroprotective action linked to the upsurge in the acetylation of non-histone proteins. So the collection of various action of HDACIs pharmacotherapy may be effective in the treatment of Alzheimer's disease. $^{6}$

\section{(III). Treatment of Epilepsy}

Currently, two classes of drugs are under investigation to be used in the epigenetic pharmacotherapy. ${ }^{6,9,11,12}$ They include DNA methyltransferase inhibitors and histone deacetylase inhibitors (HDACis). 5-azacytidine (Vidaza) is the first DNMTi approved to be used in epigenetic pharmacotherapy, whereas suberoylanilide hydroxamic acid (Zolinza/Vorinostat) was approved as HDACi. ${ }^{6,911,12}$ Also, valproic acid also possesses mild HDAC inhibiting activity; likewise, ketogenic diet with low fat, low carbohydrate, and high protein content is known to inhibit gene methylation. Overall, these drugs inhibit gene methylation but at the same time promote gene as well as histone acetylation. ${ }^{6,9,11,12}$ Another drug is called imipramine which is a class of tricyclic antidepressant, it relieves epigenetically induced stress, suppress gene methylation and induce histone acetylation. ${ }^{6,9,11,12}$ It is imperative to note that, through a clear understanding of epigenetic variations, dormant somatic cells inherited from the mother can be reactivated via nuclear transplant. ${ }^{6,911,12}$ Also, stem cells in a cancer patient can be modified epigenetically, oncogenes that promote mutagenesis can be targeted and silenced. Finally, imprinted cancer suppressing cells that were silenced can be reactivated. ${ }^{2,6,14}$

\section{(IV). Treatment of Depression}

Epigenetic variations due to gene methylation encoding serotonin transporter promoter or inhibition of neurogenesis during infancy could lead to psychiatric disorders such as depression, schizophrenia, and bipolar disorders during adulthood. ${ }^{6,9}$ Therefore, histone protein deacetylation, a reaction catalysed by histone deacetylase (HDAC) enzymes is among the possible causes. ${ }^{6,9}$ Drugs such as imipramine (a tricyclic antidepressant) which have HADC inhibitor activity and zebularine which is a transition state analogue inhibitor of cytidine deaminase could be used to treat or reverse depression caused by silencing of gene encoding serotonin transporter. ${ }^{6,9,19}$ Imipramine inhibits DNA deacetylation while zebularine inhibit DNA methylation and tumour growth both in vitro and in vivo. The overall action is to turn the genes on and favour serotonin transportation, hence the antidepressant action. ${ }^{17}$ The zebularine acts via inhibition of DNA methylation by specifically improving BDNF mRNA expression. ${ }^{6,9,16,19}$ The primary aim of using HADCis in the pharmacotherapy of depression or cancer is to reactivate SLC22A1, NR3C1, BDNF, tumour suppressor genes, and metastasis inhibitory genes and so on. ${ }^{6,9,16,19}$ Thus, suberoylanilide hydroxamic acid, SAHA (Vorinostat) is another HADCi approved in the treatment of cutaneous T-cell lymphoma. ${ }^{6,9,19}$ In an additional investigation, a valproic acid which is an effective HADCi that can be used as a mood stabilizer as well as an anticonvulsant. The studies elucidated earlier signify that epigenetic modifications could be reversed or resolved through pharmacotherapy. ${ }^{6,9}$ Markedly, apart from the impact of epigenetic changes in hippocampal and non-hippocampal brain neurogenesis during infancy, it could also affect adult and contribute to the development of psychiatric disorders at both stages. ${ }^{6,9}$ Furthermore, in 2007, sodium butyrate which is another potent HDACi has shown promise in the 
pharmacotherapy of depression in an animal model. ${ }^{6,9}$ To have effective epigenetic pharmacotherapy with DNMTs and HDACis in the future, the drug selectivity in terms of mechanism of action, brain penetrability, and drug toxicity should be properly studied. ${ }^{6,9}$ Currently, the DNMTs and HDACis used have a wide range of cytotoxicity, immune modulation and mode of actions. ${ }^{6,9}$ Interestingly, the discovery of benzamide HDAC inhibitor called MS-275 (Entinostat) which has shown selective activity for a specific HDAC, namely HDAC 1 and also ability to cross the blood-brain barrier. This drug has demonstrated selective activity by silencing the oncogenes while sparing the tumor suppressing genes. ${ }^{6,9}$ In the recent future, it is important to consider personalization of epigenetic pharmacotherapy. It could be realized by taking the individual patient DNA methylation code into consideration before prescribing epigenetics treatments. ${ }^{6,9}$ Equally, this will help in specifying the pharmacotherapy by knowing which particular gene is turned 'OFF' or 'ON' before initiating the drug treatment.

\section{(V). Treatment of Diabetes Mellitus}

It was established that type 1 Diabetes Mellitus involves autoimmune damage to pancreatic beta cells which may be as a result of the release of cytotoxic cytokines such as IL-1, IL-6, and IFN $\Upsilon$ and so on. Interestingly, HDACIs drugs act via stoppage of cytokines induced beta cells destructions and beta cells apoptosis. ${ }^{6,23}$ Moreover, the increase in iNOS in rat's primary islet cells and mouse macrophages in an in vivo experiment was achieved via inhibition of IL-6 by HDACI Vorinostat. ${ }^{6,23}$ Accordingly, the cytokines facilitated immune reactions and inflammatory responses that lead to the destruction of beta cells in Type 1 Diabetes Mellitus can be reserved by HDACIs drugs. ${ }^{6,23}$ Diabetes Mellitus also can be as a result of misregulation of autophagy. Also, the drugs used in pharmacotherapy of epigenetically induced disorder such as HDACIs has shown promise in protecting beta cells apoptosis by correcting the abnormal autophagy. ${ }^{6,23}$ The autophagy is a mechanism of cell loss through recycling intracellular components that have been produced by the pathological pathways. The GK enzymes play a vital in $\beta$-cells regeneration that may be silenced by hypermethylation. ${ }^{6,23}$ Since diabetes could result from the $\beta$-cell damage leading to the hypoinsulinemia and hyperglycemia, it could be treated through the administration of specific GK enzyme activator. ${ }^{23}$

\section{CONCLUSION}

As knowledge of epigenetics is growing, more and more answers are revealing with regards to genetically related diseases. In the recent past, several diseases such as Cancer, Diabetes, Obesity, Alzheimer's disease, Epilepsy, Depression and other Neurological disorders were only linked to genetic polymorphism and gene mutations. Later, it was discovered that many diseases are due to the epigenetic changes which only affect the DNA phenotypically. With the advancement made in the field of epigenetics, pharmacogenomics, and pharmacogenetics, the diagnoses and the treatments of aforementioned diseases become more rewarding. Through targeting the epigenetic markers, many diseases can be prevented, diagnose or treated systematically. The landmark achievement involves the possibility of silencing the oncogenes that are cancer provoking genes and possible reactivation of cancer suppression genes that battle with and repress the tumour cells proliferation. Besides these, more genes that are linked to other diseases apart from cancer can be identified, silenced or activated in order to resolve the disease aetiology. The discovery of drugs that can modify epigenetic markers such as DNMT enzymes inhibitors, or HDAC inhibitors have tremendously contributed to success in the prevention, diagnoses, and pharmacotherapy of many epigenetically induced diseases.

\section{ABBREVIATIONS}

OGG: 8-oxoguanine DNA glycosylase 1; BDNF: Brainderived neurotrophic factor; BRCA1 \& BRCA2: Breast cancer 1 and 2; CRAT: Carnitine O-acetyltransferase; F3: Coagulating factor 3; CDKN3: Cyclin-dependent kinase inhibitor 3; CPG: Cytosine-Phosphate-Guanine; DNA: Deoxyribonucleic acid; DNMT: DNA methyltransferase; ERE: Oestrogen response element; GWAS: Genome wide associations study; HDAC: Histone Deacetylase; HDACIs: Histone deacetylase inhibitors; IGF2: Insulin-like growth factor II; IFN : Interferon gamma; IL-6: Interleukin 6; ICAM: Intracellular adhesion molecule; NR3C1: Neuron-specific glucocorticoid receptor; iNOS: Nitric oxide; OCT1: Organic cation transporter 1; PEMT: Phosphatidylethanolamine N-methyltransferase; PCR: Polymerase chain reaction; SNPs: Single nucleotide polymorphism; TLR2: Toll-like receptor 2.

\section{COMPETING INTERESTS}

Authors possesses no competing interest. Authors have no financial competing interest with a person, institute or company. 


\section{AUTHORS' CONTRIBUTIONS}

ARA: conception and design, or acquisition of data, or analysis and interpretation of data, drafting the manuscript or revising.

MH: conception and design, or acquisition of data, or analysis and interpretation of data, drafting the manuscript or revising, final approval of the version to be published, final approval of the version to be published Finally, all authors have read and approved the final manuscript.

\section{ACKNOWLEDGMENT}

I extend my profound gratitude to the staff of the Faculty of Medicine, University Sultan Zainal Abidin, Kuala Terengganu, Malaysia and all the staff of the Faculty of Pharmaceutical Sciences Bayero University Kano, Nigeria. This study obtains no funding.

\section{CONFLICT OF INTEREST}

None to be declared.

\section{REFERENCES}

1. Berger SL, Kouzarides $T$, Shiekhattar R, Shilatifard A. An operational definition of epigenetics. Genes Dev. 2009;23(7):781-3. http://dx.doi. org/10.1101/gad.1787609; PMid:19339683 PMCid:PMC3959995.

2. Feinberg AP. Epigenetics at the Epicenter of Modern Medicine. JAMA. 2008;299 (11):1345-50. http://dx.doi.org/10.1001/jama.299.11.1345 PMid:18349095.

3. Madrigano J, Baccarelli A, Mittleman MA, Sparrow D, Vokonas PS, Tarantini L, et al. Aging and epigenetics: Longitudinal changes in gene-specific DNA methylation. Epigenetics. 2012;7(1):63-70. http://dx.doi.org/10.4161/ epi.7.1.18749; PMid:22207354 PMCid:PMC3329504.

4. Pignatta D, Erdmann RM, Scheer E, Picard CL, Bell GW, Gehring M. Natural epigenetic polymorphisms lead to intraspecific variation in Arabidopsis gene imprinting. Elife. 2014;3:e03198. http://dx.doi.org/10.7554/eLife.03198; PMid:24994762 PMCid:PMC4115658.

5. Williams SCP. Epigenetics. Proc Natl Acad Sci 2013;110(9):3209. http://dx.doi. org/10.1073/pnas.1302488110; PMid:23417306 PMCid:PMC3587275.

6. Vishwakarma P, Kumar A, Sharma M, Garg M, Saxena KK. Histone deacetylase inhibitors: pharmacotherapeutic implications as epigenetic modifier. Int J Basic Clin Pharmacol. 2014;3(1):27-36. http://dx.doi. org/10.5455/2319-2003.ijbcp20140236.
7. Dawson MA, Kouzarides T. Cancer Epigenetics: From Mechanism to Therapy. Cell. 2012;150(1):12-27. http://dx.doi.org/10.1016/j.cell.2012.06.013; PMid:22770212.

8. Public Health Cafe. Fast facts about Epigenetics. The Center for Ecogenetics and Environmental Health. Avaialable at: https://depts.washington.edu/ceeh/ downloads/FF_Epigenetics.pdf Accessed December 1, 2015.

9. Schroeder M, Krebs MO, Bleich S, Frieling H. Epigenetics and depression: current challenges and new therapeutic options. Curr Opin Psychiatry. 2010; 23(6):588-92. http://dx.doi.org/10.1097/YCO.0b013e32833d16c1; PMid:20644477

10. Cebrian A, Pharoah PD, Ahmed S, Ropero S, Fraga MF, Smith PL, et al. Genetic variants in epigenetic genes and breast cancer risk. Carcinogenesis. 2006;27(8):1661-9. http://dx.doi.org/10.1093/carcin/bgi375 ; PMid:16501248.

11. Kobow K, Blümcke I. The methylation hypothesis: Do epigenetic chromatin modifications play a role in epileptogenesis? Epilepsia. 2011;52(Suppl 4):15-9. http://dx.doi.org/10.1111/j.1528-1167.2011.03145.x; PMid:21732935.

12. Kobow K, El-Osta A, Blümcke I. The methylation hypothesis of pharmacoresistance in epilepsy. Epilepsia. 2013;54(Suppl 2):41-7. http:// dx.doi.org/10.1111/epi.12183; PMid:23646970.

13. Schardt D. Epigenetics: It's what turns you on....and off. Nutri. Act. Health. Let. 2013;9-11. Available at: https://www.cspinet.org/nah/pdfs/article-epigenetics. pdf Accessed December 1,2015.

14. Zolk O, Fromm MF. Drug transporter regulation in tumors by DNA methylation. Genome Med. 2012;4(1):10. http://dx.doi.org/10.1186/gm309; PMid:22293136 PMCid:PMC3334558.

15. Maggert KA. Genetics: Polymorphisms, Epigenetics, and Something In Between. Genet Res Int. 2012;2012:867951.

16. Sato F, Tsuchiya S, Meltzer SJ, Shimizu K. MicroRNAs and epigenetics. FEBS. J. 2011;278(10):1598-1609. http://dx.doi.org/10.1111/j.17424658.2011.08089.x ; PMid:21395977.

17. Stahl SM. Psychotherapy as an epigenetic 'drug': Psychiatric therapeutics target symptoms linked to malfunctioning brain circuits with psychotherapy as well as with drugs. J Clin Pharm Ther. 2012;37(3):249-53. http://dx.doi. org/10.1111/j.1365-2710.2011.01301.x; PMid:22594604.

18. Furst AL, Muren NB, Hill MG, Barton JK. Label-free electrochemical detection of human methyltransferase from tumors. Proc Natl Acad Sci. 2014;111(42): 14985-9. http://dx.doi.org/10.1073/pnas.1417351111; PMid:25288757 PMCid:PMC4210341.

19. Sharma S, Kelly TK, Jones PA. Epigenetics in cancer. Carcinogenesis. 2010;31(1):27-36. http://dx.doi.org/10.1093/carcin/bgp220; PMid:19752007 PMCid:PMC2802667.

20. Zeisel SH. Gene response elements, genetic polymorphisms and epigenetics influence the human dietary requirement for choline. IUBMB Life. 2007;59(6):380-7. http://dx.doi.org/10.1080/15216540701468954; PMid:17613168 PMCid:PMC2430110.

21. Kullmann K, Deryal M, Ong MF, Schmidt W, Mahlknecht U. DNMT1 genetic polymorphisms affect breast cancer risk in the central European Caucasian population. Clin Epigenetics. 2013;5(1):7. http://dx.doi.org/10.1186/18687083-5-7 ; PMid:23638630 PMCid:PMC3646668.

22. Park PJ. Epigenetics meets next-generation sequencing. Epigenetics. 2008;3(6):318-21. http://dx.doi.org/10.4161/epi.3.6.7249; PMid:19098449.

23. GilbertER,LiuD.Epigenetics:themissinglinktounderstanding $\beta$-celldysfunction in the pathogenesis of type 2 diabetes. Epigenetics. 2012;7(8):841-52. http://dx.doi.org/10.4161/epi.21238;PMid:22810088;PMCid:PMC3427279.

\section{SUMMARY}

- The literature review was aimed at highlighting the concept of epigenetics and basic phenotypic changes that affects the expression of normal traits from parents to the offspring. Health implications of changes in genome structure that do not affect the gene sequence but only some external modifications. These changes can be manipulated and utilized in the prevention and treatment of chronic diseases such as cancer, diabetes, Alzheimer's disease etc. The goal of modern disease treatment is proper utilization of evident based medicine and approaching the disease etiology at molecular level. This can be achieved through the thorough the knowledge of genetic modifications and its contributions to the disease pathology and drug efficacy. 